\title{
As equações de predição da taxa metabólica basal são apropriadas para adolescentes com sobrepeso e obesidade?
}

Patrícia Schneider ${ }^{1}$ e Flávia Meyer ${ }^{2}$

\section{RESUMO}

As equações de predição conhecidas podem apresentar valores de taxa metabólica basal (TMB) diferentes daqueles medidos por calorimetria indireta. Os objetivos deste estudo foram descrever a TMB, por meio de calorimetria indireta, em meninos com sobrepeso e obesidade, de 12 a 17 anos de idade, residentes em Porto Alegre, Brasil, e comparar o valor medido com os valores de TMB estimados por equações de predição. A TMB foi medida por calorimetria indireta, pela manhã, em 35 voluntários, sob condições padronizadas de jejum, repouso e ambiente. A média ( \pm desviopadrão) da TMB medida foi de 1.900,5 $\pm 248,8 \mathrm{kcal}$ em 24 horas. A estimativa da TMB por equações foi significativamente maior, em três das quatro equações $(6,5$ a 9,5\%), do que a TMB medida ( $p<$ $0,05)$. Os dados evidenciaram que as equações de predição não são adequadas para estimar a TMB nos meninos com sobrepeso e obesidade avaliados. O emprego dessas equações, na maioria dos casos, pode superestimar os requerimentos energéticos para meninos com características semelhantes.

\section{ABSTRACT \\ Are basal metabolic rate prediction equations appropriate for overweight and obese adolescents?}

The known predictive equations can present different values for basal metabolic rate (BMR) compared to those measured through indirect calorimetry. The objective of this study was to describe $B M R$ through indirect calorimetry of overweight and obese boys (with ages between 12 and 17 years old) living in Porto Alegre, Brazil, and to compare the measured value with values estimated by predictive equations. Thirty-five volunteers had their BMR measured through indirect calorimetry in the morning, under standard conditions of fasting, rest and environment. The average ( \pm standard deviation) of measured BMR was of $1,900.5 \pm 248.8 \mathrm{kcal} / 24$ hours. Estimated BMR were significantly greater, in three of four equations (6.5 to 9.5\%), than measured BMR $(p<0.05)$. These results show that predictive equations are not suitable to estimate $B M R$ in these groups of overweight and obese boys. The use of estimated BMR can lead, in most cases, to an overestimation of energy requirements for boys with similar characteristics.

1. Mestranda em Ciências do Movimento Humano na Escola de Educação Física da Universidade Federal do Rio Grande do Sul, Porto Alegre, RS, Brasil.

2. Professora na Escola de Educação Física da UFRGS. Doutora pela McMaster University, Canadá.

Recebido em 24/2/05. 2a versão recebida em 16/3/05. Aceito em 4/5/05.

Endereço para correspondência: Profa. Patrícia Schneider, Escola de Educação Física da UFRGS, Rua Felizardo, 750, B. J ardim Botânico - 90690200 - Porto Alegre, RS. Tel.: (51) 9654-8762. E-mail: schneiderpatricia@ yahoo.com.br

\begin{abstract}
Palavras-chave: Metabolismo basal. Valor preditivo. Calorimetria. Gasto energético. Key words: Basal metabolism. Predictive value. Calorimetry. Energy expenditure.

Palabras-clave: Metabolismo basal. Valor predictivo. Calorimetría. Gasto energético.
\end{abstract}

\section{RESUMEN}

\section{¿Las ecuaciones de prediccion de la tasa metabolica basal son apropiadas para adolescentes con sobrepeso y obesidad?}

Las ecuaciones de predicción conocidas pueden presentar valores de tasa metabólica basal (TMB) diferentes de aquellos medidos por calorimetria indirecta. Los objetivos de este estudio fueron describir la TMB, por medio de calorimetría indirecta, en chicos con sobrepeso y obesidad, de 12 a 17 años de edad residentes en Porto Alegre, Brasil, y comparar el valor medido con los valores de TMB estimados por ecuaciones de predicción. La TMB fué medida por calorimetria indirecta, por la mañana, en 35 voluntarios, sobre condiciones padronizadas de ayuno, reposo y ambiente. La media $( \pm D P)$ de la TMB medida fué 1.900,5 $\pm 248,8 \mathrm{kcal}$ en 24 horas. La estimativa de la TMB por ecuaciones fué significativamente mayor, en tres de las cuatro ecuaciones (6,5 a 9,5\%), de que la TMB medida $(p<0,05)$. Los datos evidenciaron que las ecuaciones de predicción no son adecuadas para estimar la TMB en los niños con sobrepeso y obesidad evaluados. El empleo de esas ecuaciones, en la mayoría de los casos, puede superestimar los requerimentos energéticos para chicos con características semejantes.

\section{INTRODUÇÃO}

O gasto energético diário compreende o dispêndio basal, o efeito térmico dos alimentos e o dispêndio da atividade física(1). O dispêndio basal representa 60 a 75\% do custo energético diário e inclui a energia gasta com a manutenção das funções vitais do organismo. A energia gasta para a atividade física representa 15 a $30 \%$ do dispêndio energético diário e varia com o nível de atividade física do indivíduo(2).

A calorimetria indireta $(\mathrm{Cl})$ é um método que determina as necessidades nutricionais a partir do consumo de oxigênio e da produção de gás carbônico obtidos através do ar inspirado e expirado pelos pulmões ${ }^{(3,4)}$. A denominação indireta indica que a produção de energia, diferentemente da calorimetria direta, que mede a transferência de calor do organismo para o meio ambiente, é calculada a partir dos equivalentes calóricos do oxigênio consumido e do gás carbônico produzido. Assim, calcula-se a quantidade total de energia produzida, utilizando o oxigênio consumido na utilização da oxidação dos substratos energéticos e o gás carbônico que é eliminado pela respiração(5). É um método prático para identificar a natureza e a quantidade dos substratos energéticos que estão sendo metabolizados pelo organismo(5).

A Cl exige alguns cuidados em relação ao ambiente, ao indivíduo e a aspectos técnicos. O ambiente deve ser silencioso, com pouca iluminação e estar numa temperatura confortável, para evi- 
tar alterações causadas por frio ou ansiedade. Para a taxa metabólica de repouso, o indivíduo deve estar em repouso há pelo menos 30 min e observar um jejum de duas a três horas ${ }^{(6,7)}$. J á o metabolismo basal requer maior cuidado, com jejum de 12 horas e devendo ser medido após acordar, pela manhã.

Os estudos mostram uma duração de coleta de gases inspirados e expirados de mais ou menos $20 \mathrm{~min}$, sendo uma média realizada no intervalo de 10min mais constantes ${ }^{(8,9)}$. O gasto energético medido nesse intervalo é extrapolado para 24 horas e é considerado representativo do dispêndio energético basal diário $^{(6,10,11)}$

O dispêndio energético pode ser medido por $\mathrm{Cl}$ ou, de forma mais simples, estimado por equações preditivas. Em 1985, a Organização M undial da Saúde passou a recomendar que os requerimentos energéticos fossem baseados na medição do gasto energético ${ }^{(12)}$. Considerando que na maioria das vezes não é possível medir a taxa metabólica basal (TMB), recomendou-se o uso internacional de equações de predição da TMB, modificadas de uma compilação de dados realizada por Schofield(13) $(1985)$. Alguns estudos têm sido realizados com diferentes grupos(14-17) e têm demonstrado que essas equações fornecem estimativas elevadas de TMB, particularmente para os residentes nos trópicos. Essas diferenças podem ser explicadas pelo fato de essas equações originarem-se, na maioria, de amostras das populações norte-americana e européia, que podem apresentar características diferenciadas de composição corporal e vivem em ambientes distintos.

A equação de Harris e Benedict(18) $(1919)$, que é uma das mais utilizadas e conhecidas, estima o dispêndio de repouso com uma precisão de cerca de $10 \%$ em 80 a $90 \%$ dos indivíduos saudáveis $^{(7)}$. Ao se empregar uma equação preditiva, é importante saber a população da qual ela foi obtida e os fatores que afetam e alteram a capacidade preditiva(19).

Henry e Rees ${ }^{(17)}$ (1991) reuniram dados de TMB de residentes em trópicos e criaram equações específicas para essas populações. Apesar de essas equações fornecerem estimativas menores quando comparadas com as obtidas pelas equações da FAO/ WHO/UNU (12) (1985), os valores por elas estimados parecem, ainda, superestimar a TMB em regiões tropicais $(14,20)$.

Um estudo em universitárias, no Rio de J aneiro ${ }^{(14)}$, demonstrou que as equações de predição superestimavam a TMB entre 7 e $19 \%$; outro estudo com crianças não-obesas demonstrou que nenhuma das cinco equações de predição foi capaz de estimar o gasto energético(21).

É necessário que mais estudos sejam realizados nos vários segmentos da população brasileira para que se possam validar ou propor equações adequadas de predição da TMB. Como a $\mathrm{Cl}$ é um teste caro que requer cuidados, a estimativa acurada do dispêndio e da ingesta calórica para o planejamento alimentar seria facilitada por meio de equações de predição, bem como seria muito importante para esse grupo que tem o objetivo de perder peso(21).

Pesquisas comparando a TMB medida com a TMB predita por equações de predição em meninos adolescentes obesos são escassas ${ }^{(22)}$. Portanto, o presente estudo teve por objetivos descrever a TMB por meio da $\mathrm{Cl}$, em um grupo de meninos com sobrepeso e obesidade, e comparar a TMB medida com a TMB estimada pelas equações de FAO/WHO/UNU(12) (1985), Harris e Benedict(18) (1919), Henry e Rees ${ }^{(17)}$ (1991) e Schofield(13) (1995).

\section{MÉTODOS}

A amostra compreendeu 35 meninos com sobrepeso e obesidade, residentes em Porto Alegre, Rio Grande do Sul, estudantes de um colégio da cidade, com idade entre 12 e 17 anos. Destes, 24 eram púberes e 11, pós-púberes, segundo Tanner ${ }^{(23)}$ (1962). A participação dos voluntários só ocorreu mediante a assinatura de termo de consentimento dos mesmos e de um dos pais ou responsáveis. O projeto foi aprovado pelo Comitê de Ética em Pes- quisa do Hospital de Clínicas de Porto Alegre (HCPA), que emitiu parecer favorável à sua execução, sob o número 03-497.

O percentual de gordura corporal foi medido por meio do exame de Absortometria Radiológica de Dupla Energia (DEXA), da marca Lunar, modelo DPX-L, com o programa pediátrico SmartScan, versão 4.7c. A gordura corporal foi utilizada para avaliar se os voluntários apresentavam sobrepeso ou obesidade e para as correlações com a TMB.

A coleta da TMB foi realizada por meio da calorimetria indireta. Para isso, foi utilizado um analisador de gases da marca MedGraphics Cardiorespiratory Diagnostic Systems, modelo CPX/D da Medical Graphics Corporation. Esse aparelho coleta amostras de gases através de uma máscara ou de um bocal e um oclusor nasal. As coletas foram realizadas entre sete e nove horas, durante a segunda quinzena de fevereiro e a primeira de março de 2004. Os participantes compareceram ao local no dia e na hora agendados; foi verificada a adesão ao protocolo para medição da TMB, que incluía jejum de no mínimo 12 horas, oito horas de sono na noite anterior à medição e manutenção das atividades cotidianas, evitando-se atividades físicas intensas na véspera. Para avaliação da massa corporal, foi utilizada uma balança de equilíbrio da marca Filizola, com resolução de $0,1 \mathrm{~kg}$. Os meninos foram pesados com camiseta e bermuda, e descalços. Para medir a estatura, utilizouse um estadiômetro constituído por escala métrica com resolução de $1 \mathrm{~mm}$, em que foi medida a estatura do indivíduo de pé e descalço.

A TMB foi coletada em sala tranqüila, com condições adequadas: temperatura ambiente controlada, baixa luminosidade e sem ruídos. Inicialmente, o teste era explicado ao voluntário, que permanecia 20min deitado em repouso. Após, uma máscara era fixada no rosto do voluntário e conectada ao calorímetro. O consumo de oxigênio $\left(\mathrm{VO}_{2}\right)$ e a produção de gás carbônico $\left(\dot{\mathrm{V}} \mathrm{CO}_{2}\right)$ foram medidos durante 15 a $25 \mathrm{~min}$ com o voluntário permanecendo deitado e imóvel. Esse tempo foi escolhido de acordo com o padrão dos dados apresentados no momento do teste, isto é, se havia pouca variação nos dados, o tempo de medição era menor; se havia maior variação, o tempo de medição aumentava.

A TMB medida por meio da Cl foi utilizada na comparação com os valores obtidos pelas equações de predição de TMB (quilocalorias por dia $=\mathrm{kcal}$ em $24 \mathrm{~h}$ ) mais utilizadas, em que $\mathrm{MC}=$ massa corporal, EST = estatura e ID = idade:

1 - Harris e Benedict(18) (1919) (15 a 74 anos, masculino): 66,4730 $+(13,7516 \times \mathrm{MC})+(5,0033 \times \mathrm{EST})-(6,7550 \times$ ID $)$

2 - Schofield(13) (1985) (10 a 18 anos): $(0,074 \times$ MC) + 2,754

3 - FAO/WHO/UNU(12) (1985) (10 a 18 anos): $(0,0732 \times$ MC) + 2,72

4 - Henry e Rees ${ }^{(17)}$ (1991) (10 a 18 anos): (0,084 x MC) + 2,122

As três últimas equações apresentam o resultado em milijoules por dia (mJ /dia), sendo o resultado multiplicado por 239 para obtenção do valor em quilocalorias (kcal). Os percentuais de diferença entre os valores de TMB estimados por cada uma das equações listadas acima e o medido foram assim calculados: [(TMB estimada - TMB medida)/TMB medida] x 100.

A análise estatística incluiu o teste $t$ pareado para a comparação das diferenças entre o valor da TMB medida e os valores obtidos pelas equações de predição. O coeficiente de correlação linear de Pearson foi utilizado para descrever a correlação entre a TMB, as medidas antropométricas e a composição corporal. Calculou-se, também, o coeficiente de correlação parcial entre essas variáveis após o controle pela massa corporal. O nível de significância utilizado foi de $p<0,05$.

\section{RESULTADOS}

A média $( \pm \mathrm{dp}$ ) das características físicas, a da TMB medida e a da TMB estimada pelas equações de predição estão na tabela 1. 
TABELA 1

Características físicas e resultados da TMB medida e predita (md \pm dp)
$\mathrm{N}$

Idade (anos)

Massa corporal $(\mathrm{kg})$

Estatura $(\mathrm{cm})$

IMC

Gordura corporal (\%)

aTMB - Calorimetria indireta (kcal/dia)

Equação Harris e Benedict (kcal/dia)

Equação Schofield (kcal/dia)

Equação FAO/WHO/UNU (kcal/dia)

Equação Henry e Rees (kcal/dia)
35

$13,7 \pm 11$

$77,2 \pm 9,8$

$166,7 \pm 8,5$

$27,7 \pm 2,0$

$36,6 \pm 8,8$

$1.900,5 \pm 249,8$

$1.870,2 \pm 169,2$

$2.024,2 \pm 173,7^{*}$

$2.001,3 \pm 171,8^{*}$

$2.057,7 \pm 197,2^{*}$
* > TMB medida $(p<0,05)$; ${ }^{\text {a TMB }}=$ taxa metabólica basal.

A equação de Harris e Benedict foi a única que não demonstrou diferenças entre a TMB medida e a predita $(p<0,05)$. As outras equações superestimaram a TMB $(p<0,05)$, sendo a de Henry e Rees a que mais superestimou (9,5\%), seguida da TMB estimada pela equação de Schofield $(7,7 \%$ ) e da equação da FAO/WHO/UNU $(6,5 \%)$. Embora três das quatro equações empregadas superestimassem a TMB, houve subestimativa da TMB com essas mesmas equações em alguns casos: $28,6 \%$ dos voluntários tiveram valores estimados de TMB pela equação de Schofield menores do que os valores medidos, $31,4 \%$ com a equação da FAO/WHO/UNU, e $25,7 \%$ com a equação de Henry e Rees.

A TMB medida apresentou correlação significativa com as seguintes medidas antropométricas e de composição corporal: idade $(r=0,55)$, massa corporal $(r=0,49)$, estatura $(r=0,60)$ e gordura corporal $(r=-0,52)$. As correlações de segunda ordem, controladas pela massa corporal, com significância estatística entre a TMB e as medidas antropométricas e de composição corporal, tenderam a diminuir: idade $(r=0,45)$, estatura $(r=0,40)$, IMC $(r=-0,41)$, gordura corporal $(r=-0,48)$.

\section{DISCUSSÃO}

A média de TMB medida em adolescentes obesos neste estudo foi de $1.900 \mathrm{kcal} / \mathrm{dia}$. Esse valor foi comparado com quatro equações de predição e apenas uma delas, a de Harris e Benedict, não diferiu significativamente da TMB medida $(p>0,05)$. Esse resultado não surpreende, já que essas equações de predição não tiveram origem com grupo de adolescentes obesos, mas sim com jovens fisicamente ativos $(45 \%)$ e adultos ${ }^{(12,13)}$. Assim, é preciso cautela quando as equações de predição de TMB são extrapoladas para adolescentes.

Também é necessário cuidado na comparação da TMB entre crianças e adolescentes obesos e não-obesos, já que esses valores parecem não diferir apenas quando ajustados pela massa corporal magra (MCM), que é o seu melhor preditor e responde por até $80 \%$ da sua variância(22,24,25). Apenas um estudo(22) encontrou maiores valores de TMB em obesos comparando com não-obesos, mesmo quando ajustados pela MCM.

Muitos estudos têm demonstrado superestimativa da TMB pelas equações de predição. Em um estudo de McDuffie et al.(21) (2004), com 502 meninos e meninas entre seis e 11 anos, nãoobesos, brancos e negros, nenhuma das cinco equações de predição mostrou estimativa acurada do gasto energético medido por calorimetria indireta. Outro estudo(26) mostrou que as equações de predição não são apropriadas para estimar a TMB acuradamente em crianças e adolescentes do sexo feminino, não-obesas, pois, cinco das equações superestimaram a TMB, entre elas as de Harris e Benedict, FAO/WHO/UNU e Schofield.

Dados de Spur et al. ${ }^{(27)}$ (1992) também superestimaram a TMB pela equação de Schofield, em meninos mestiços não-obesos, mas não em meninas.
Um estudo de Dietz et al.(28) (1991) realizado em adolescentes mostrou valores similares de TMB medida por $\mathrm{Cl}$, comparados com os da equação de FAOMHO/UNU. Porém, Henry e Rees(17) (1991) demonstraram que essa equação superestima a TMB de crianças e adolescentes que vivem nos trópicos, corroborando os dados do presente estudo. Além desse estudo, Maffeis et al.(29) (1993), que analisaram pré-púberes (33 obesos e 97 não-obesos), demonstraram que as equações de predição superestimaram a TMB, ainda mais em obesos do que em não-obesos, estando entre elas a de FAO/WHO/UNU.

Ainda comparando a predição da TMB pela equação de FAO/ WHO/UNU, o estudo de Van Mil et al. (22) (2001) com adolescentes holandeses demonstrou a sua superestimativa em meninos obesos e a subestimativa em não-obesos. Nesse estudo, foram maiores os valores da taxa metabólica de repouso de obesos comparados com os de não-obesos de ambos os sexos (1.997 vs. 1.535kcal/ dia) e os de meninos obesos comparados com os de meninas obesas (2.129 vs. $1.839 \mathrm{kcal} / \mathrm{dia})$.

As equações de predição em adultos superestimam a TMB medida em 2,2 a 13,5\% $(14,15,17,20,25)$. No estudo presente, os resultados desse percentual variaram dentro dessa faixa, indo de 6,5 a $9,5 \%$.

O estudo de Wahrlich e Anjos(16) (2001), o qual ocorreu na mesma cidade do presente estudo, com mulheres de 26 anos nãoobesas, apresentou maiores valores percentuais de superestimativa das equações de Schofield e FAO/WHO/UNU comparados com os do presente estudo, sendo 12,5 e $13,5 \%$ vs. 7,7 e $6,5 \%$, respectivamente. Em relação às subestimativas, a equação de $\mathrm{FAO} /$ WHO/UNU subestimou os valores de TMB de $20 \%$ das mulheres, comparados com 31,4\% dos meninos do presente estudo.

Foi novidade a adequação da estimativa da TMB pela equação de Harris e Benedict em adolescentes obesos, já que, em outro grupo, como de mulheres norte-americanas, o próprio Benedict constatou que a equação sistematicamente fornecia valores maiores do que a TMB medida e recomendou que as estimativas fossem reduzidas em $5 \%{ }^{(25)}$. Talvez essa equação tenha sido a que mostrou os melhores resultados por utilizar a estatura e a idade, 0 que não ocorre nas outras equações.

Os valores da TMB encontrados nesse estudo são similares àqueles encontrados na literatura, com protocolo similar. Nesses estudos, Molnar e Schutz ${ }^{(24)}$ (1997) e Stensel et al.(30) (2001) apresentaram 1.727 e $1.702 \mathrm{kcal} / \mathrm{dia}$, respectivamente, em grupos de meninos adolescentes obesos da mesma faixa etária.

O estudo de Wong et al. ${ }^{(26)}$ (1996) apresentou TMB de 1.350kcal/ dia para crianças e adolescentes do sexo feminino, não-obesas. Esse valor está abaixo do medido neste estudo, concordando com dados de Van Mil et al.(22) (2001), que apresentaram menores valores de TMB para meninas comparados com os de meninos e para não-obesos comparados com obesos.

No presente estudo, as medidas antropométricas foram significativamente correlacionadas com a TMB, com coeficientes de correlação variando entre 0,49 e 0,60. Os valores dos coeficientes de correlação parcial entre a TMB e as medidas de idade, estatura, IMC e gordura corporal controlando-se pela massa corporal tenderam a diminuir, o que indica que as correlações preexistentes entre TMB e as outras medidas corporais sejam apenas um reflexo da massa corporal.

Uma limitação deste estudo é a amostra reduzida e apenas do sexo masculino. Para estudos futuros, sugerimos a ampliação da amostra e realização do mesmo com meninas obesas, objetivando a confecção de equações de predição para esses grupos, já que existem diferenças de gênero na avaliação do metabolismo basal.

A inadequação das equações de predição pode acarretar erros na estimativa da necessidade energética de populações. Para os adolescentes obesos avaliados no presente estudo, a necessidade energética obtida pela multiplicação da TMB pelo fator de atividade (nível de atividade física) de 1,56, que é o valor recomendado 
para a atividade leve(12), seria de $2.965 \mathrm{kcal} / \mathrm{dia}$, quando utilizada a TMB medida, e de 3,210kcal/dia, quando utilizada a TMB estimada pela equação de Henry e Rees. A utilização dessa equação de predição levaria a uma superestimativa média de aproximadamente 250kcal/dia, resultando em um balanço energético positivo, o que seria inaceitável para esse grupo, com alta gordura corporal. A manutenção de um balanço energético positivo poderia agravar essa condição, levando a uma prevalência ainda maior de obesidade. O mesmo poderia ser esperado para as demais equações de predição, pois todas forneceram, em média, estimativas significativamente maiores do que a TMB medida.

\section{AGRADECIMENTOS}

Agradecemos ao CNPq, à Escola de Educação Física da UFRGS e, especialmente, à Clínica de Densitometria Óssea Clinodens-RS.

Todos os autores declararam não haver qualquer potencial conflito de interesses referente a este artigo.

\section{REFERÊNCIAS}

1. Houssay BA. El intercambio material y energético del organismo. In: Fisiología humana. Buenos Aires: Librería El Ateneo Editorial, 1969;487-502.

2. Astrand PO, Rodahl K. Textbook of work physiology. New York: McGraw-Hill Book, 1970.

3. Ferrannini $\mathrm{E}$. The theoretical bases of indirect calorimetry: a review. Metabolism 1988;37:287-301.

4. Branson RD. The measurement of energy expenditure: instrumentation, practical considerations, and clinical application. Respir Care 1990;35:640-59.

5. Diener J RC. Calorimetria indireta. Rev Assoc Med Brasil 1997;43:245-53.

6. Mullen J L. Indirect calorimetry in critical care. Proc Nutr Soc 1991;50:239-44.

7. Dickerson RN, Vehe KL, Mullen J L, Feurer ID. Resting energy expenditure in patients with pancreatitis. Crit Care Med 1991;19:484-90.

8. Isbell TR, Klesges RC, Meyers AW, Klesges LM. Measurement reliability and reactivity using repeated measurements of resting energy expenditure with a face mask, mouthpiece, and ventilated canopy. J PEN J Parenter Enteral Nutr 1991; 15:165-8.

9. Stokes MA, Hill GL. A single, accurate measurement of resting metabolic expenditure. J PEN J Parenter Enteral Nutr 1991;15:281-7.

10. Van Lanschot JJ, Feenstra BW, Vermeij CG, Bruining HA. Calculation versus measurement of total energy expenditure. Crit Care Med 1986;13:981-5.
11. McClave SA. Effective utilization of indirect calorimetry during critical care. Intensive Care World 1992;9:194-200.

12. FAO/WHO/UNU. Energy and protein requirements. Geneva: World Health Organization, 1985. [WHO Technical Report Series, 724].

13. Schofield WN. Predicting basal metabolic rate, new standards and review of previous work. Hum Nutr Clin Nutr 1995;39C:5-41.

14. Cruz CM, Silva AF, Anjos LA. A taxa metabólica basal é superestimada pelas equações preditivas em universitárias do Rio de J aneiro, Brasil. Arch Latinoam Nutr 1999;49:232-7.

15. Piers LS, Diffey B, Soares MJ, Frandsen SL, McCormack LM, Lutschini MJ , et al. The validity of predicting the basal metabolic rate of young Australian men and women. Eur J Clin Nutr 1997;51:333-7.

16. Wahrlich V, Anjos LA. Validação de equações de predição da taxa metabólica basal em mulheres residentes em Porto Alegre, RS, Brasil. Rev Saúde Pública 2001;35:39-45.

17. Henry CJ K, Rees DG. New predictive equations for the estimation of basal metabolic rate in tropical peoples. Eur J Clin Nutr 1991;45:177-85.

18. Harris J A, Benedict FG. A biometric study of basal metabolism in man. Boston: Carnegie Institution of Washington, 1919.

19. Weissman C, Kemper M. Metabolic measurements in the critically ill. Crit Care Clin 1995;11:169-97

20. Piers LS, Shetty PS. Basal metabolic rates of Indian women. Eur J Clin Nutr 1993;47:586-91.

21. McDuffie J R, Adler-Wailes DC, Elberg J , Steinberg EN, Fallon EM, Tershakovec $A M$, et al. Prediction equations for resting energy expenditure in overweight and normal-weight black and white children. Am J Clin Nutr 2004;80:365-73.

22. Van Mil EGAH, Westertep KR, Kester ADM, Saris WHM. Energy metabolism in relation to body composition and gender in adolescents. Arch Dis Child 2001; 85:73-8.

23. Tanner J M. Growth and adolescence. Oxford: Blackwell Scientific Publications, 1962.

24. Molnár D, Schutz Y. The effect of obesity, age, puberty, and gender on resting metabolic rate in children and adolescents. Eur J Pediatr 1997;156:378-81.

25. Wahrlich V. Taxa metabólica basal em mulheres residentes em Porto Alegre, Rio Grande do Sul [Dissertação]. Rio de J aneiro: Universidade Federal do Rio de J aneiro, 2000.

26. Wong WW, Butte NF, Hergenroeder AC, Hill RB, Stuff J E, Smith EO. Are basal metabolic rate prediction equations appropriate for female children and adolescents? J Appl Physiol 1996;81:2407-14.

27. Spurr GB, Reina J C, Hoffmann RG. Basal metabolic rate of Colombian children 2-16yr of age: ethnicity and nutritional status. Am J Clin Nutr 1992;56:623-9.

28. Dietz WH, Bandini LG, Schoeller DA. Estimates of metabolic rate in obese and nonobese adolescents. J Pediatr 1991;118:146-9.

29. Maffeis $C$, Schutz $Y$, Micciolo R, Zoccante $L$, Pinelli L. Resting metabolic rate in six-to ten-year-old obese and nonobese children. J Pediatr 1993;122:556-62.

30. Stensel DJ , Lin FP, Nevill AM. Resting metabolic rate in obese and nonobese Chinese Singaporean boys aged 13-15y. Am J Clin Nutr 2001;74:369-73. 